\title{
XVII. On the aëriform compounds of charcoal and hydrogen; with an account of some additional experiments on the gases from oil and from coal
}

\author{
Wm. Henry M.D. F.R.S.
}

To cite this article: Wm. Henry M.D. F.R.S. (1821) XVII. On the aëriform compounds of charcoal and hydrogen; with an account of some additional experiments on the gases from oil and from coal , Philosophical Magazine Series 1, 58:280, 90-98, DOI: 10.1080/14786442108652573

To link to this article: http://dx.doi.org/10.1080/14786442108652573

电 Published online: 29 Jul 2009.

Submit your article to this journal $\pi$

山 Article views: 2

View related articles 
In calculating the corrections of $\lambda$ and $S$ three places of the logarithms are sufficient.

$$
\begin{aligned}
& \mathrm{D}-d=\mathrm{l}^{\prime} \cdot 5 \\
& \text { Log. } 1^{\prime} \cdot 5, \quad 0 \cdot 176 \\
& \text { Sin S; } \quad 9 \cdot 899 \\
& \text { A.C. } \sin t, \quad 10.417 \\
& \log 3 \cdot 1, \quad \overline{0 \cdot 492} \\
& \lambda=48^{\circ} 53^{\prime} \cdot 3 \\
& \frac{-3 \cdot 1}{48 \quad 50^{\prime \cdot 2}} \\
& \text { Lng. I'.5 } 0.176 \\
& \text { Cos S, 9.785 } \\
& \text { Tan } \lambda, \quad 10.059 \\
& \text { A.C. } \sin t, 10.417 \\
& \operatorname { l o g } 2 \cdot 7 \longdiv { 0 . 4 3 7 } \\
& \mathrm{~S}=52^{\circ} 28^{\prime} \\
& \begin{array}{r}
+\quad 2 \cdot 7 \\
\hline 5230^{\prime} \cdot 7
\end{array}
\end{aligned}
$$

true latitude.

true hor. angle of $\mathrm{M}$. $\mathrm{T}$.

The method that has been explained requires only the easy lemma for compriting the arcs $Z D$ and $D O$, and the rules for solving right-angled spherical triangles; and it is an advantage that every step is the calculation of some part of the figure, by which circumstance the memory is assisted. The process here followed is also preferable to the other methods in leading to the determination of the problem, or in pointing out which of the two possible solutions is the true one, when this can be done. In the extensive Nautical Tables published by the late Mr. Mendoza, there is one for assisting the direct solution of this problem. It contains the base, and likewise the angle at the hase, of the isosceles triangle A P B formed by the two circles of declination. A similar table that should contain the perpendicular $\mathrm{PO}$ of the same triangle, and likewise half the base $\mathrm{AO}$, or rather the sine and co-sine of $\mathrm{AO}$, would render the preceding method by far the shortest of any hitherto proposed. But the use of such tables is not free from objection, and ought not to be adopted unless a great advantage is gained.

August 6,1821 .

J. IYORY.

XVII. On the aëriform Compounds of Charcoal and Hydrogen; with an Account of some additional Experiments on the Gases from Oil and from Coal. By WM. Henry, M.D. F.R.S.

[From the Transactions of the Royal Society.]

T $\mathrm{T}$ experiments on the aerriform compounds of charcoal and hydrogen, described in the following pages, are supplementary to a Memoir on the same class of bodies, which the Royal Society did me the honour to insert in their Transactions for $1508^{*}$, as well as to other papers on the same subject, which have been published in Mr. Nicholson's Journal, and in the Memoirs of the Manchester Society. Of these essays, I beg leave to offer a very

* See Phil. Mag. vol. xxxii. p. 277. 
brief recapitulation, with the view merely of connecting them with what is to follow.

In the first of these essays (Nicholson's Journal, Svo, June, 180.5), I detailed a series of experiments on the gases obtained by the destructive distillation of wood, peat, pit-coal, oil, wax, \&c., from which it appeared that the fitness of those gases for artificial illumination was greater, as they required for combustion a greater proportional volume ofoxygen; and that the gases generated from different inflammable bodies, or from the same inflammable substance under different circumstances, are not so many distinct species, whicb under such a view of the subject would be almost infinite in number, but are inixtures of a few well known gases, chiefly of carburetted hydrogen with variable proportions of olefiant, simple hydrogen, sulphuretted hydrogen, carbonic acid, carbonic oxide, and azotic gases; and that the elastic fuids obtained from coal, oil, \&ic. have probably, in addition to these, an inflammable vapour diffused through them when recent, which is not removed by passing them through water*. In the same paper I explained certain anomalies that appear in the experiments of the late Mr. Cruickshank, of Woolwich, which are not at all chargeable as errors upon that excellent chemist, and could only be elucidated by further investigation of the gases to which they relate. Of his labours it would be unjust, indeed, to speak in any terms but those of approbation, for they may fairly be considered as the foundation of most that is now known respecting this species of aëriform bodies. To Mr. Dalton, also, we are indebted for an accurate acquaintance with carburetted hydrogen gas, and for much information that is valuable in assisting us to, judge of the composition of mixed combustible gases, by the phænomena and results of firing them with oxygen $\uparrow$.

In the second Mernoir (Philosophical Transactions, 1808), I described a series of experiments on the gases obtained from several different varieties of pit-coal, and from the same kind of coal under different circumstances. Various species of that mineral were found to yield aëriform products, differing greatly in specific gravity, combustibility, and illuminating power; the cannel coal of Wigan, in Lancashire, being best adapted to the purpose, and the stone-coal of South Wales the least so. In decomposing any one species of coal, the gaseous fluids were ascertained not to be of uniform quality throughout the process, but to vary greatly at different stages; the heavier and more combustible gases coming over first, and the lighter and less combustible afterwards. By subsequent experiments on the gases obtained from coal on the large scale of manufacture, it was found

* Nichoison's Journal, 8vo. xi. 72.

$\uparrow$ New Systen of Chemical Philowophy, passim. 
that a imilar decline in the value of the products takes place, but not to the same extent, owing, probably, to the greater uniformity of temperature which is attainable in large operations *.

On the practical conclusions, which it was the object of the last mentioned Essay to establish, I forbear to dwell, because they are unconnected with my present purpose, which is limited to the chemical constitution of these compound gases, and to the methods of separating them accurately from each other. The view of their nature and composition, which was taken in the first Essay, was opposed by those able philosophers M. Berthollet, and Dr. Murray, of Edinburgh, who both contended for greater latitude as to the proportions in which hydrogen and charcoal are capable of uniting, and considered these proportions indeed as subject to no limitation. The facts, however, which have since been multiplied in this, as well as in other departments of chemistry, tending to prove that bodies capable of energetic combination unite in a few definite proportions only, leave little doubt that the same law holds good with respect to the compounds of hydrogen and charcoal. Not that it is meant that the known compounds of those elements are the only possible ones; for others will probably be discovered, which will still be found conformable to the general law, that when one body comlines with another in differsnt proportions, the greater proportions are multiples of the less by an entire number.

A different view of the subject has lately been taken by the ingenious author of the Bakerian Lecture, published in the Philosophical Transactions for 1820 . In that paper, Mr. Brande has endeavoured to prove, that the gas called light carburetted hydrogen, or simply carburetted hydrogen, or hydro-carburet, is not entitled to be considered as a distinct species; that the only aëriform compound of charcoal and hydrogen, which is with certainty known to exist, is the gas called olefiant, or bi-carburetted hydrogen; and that the gases evolved by heat from coal and oil, are in fact nothing more than mixtures of olefiant and simple hydrogen gases in various proportions.

In assuming, in the first Essay, the existence of light carburetted hydrogen as a definite compound, characterized by its renuiring, for the complete combustion of each volume, two volumes of oxygen, and giving one volume of carbonic acid, I relied on the sole authority of Mr. Dalton; for the gas of marshes, though before known to be inflammable, har not been subjected to accurate examination by any other chemist. Mr. Cruickshank, indeed, speaks of it as " pure hydro-carbonate $\uparrow ; "$ but since he classes it in that respect with the gas obtained by the destructive

" Manchester Society's Memoirs, New Serics, vol, iij.

$\uparrow$ Nicholson's Journal, 4to. vol. F. p. 6. 
distillation of camphor, from which it differs essentially in composition, it is plain that he was not correctly acquainted with the properties of pure carburetted hydrogen. Previously to the second set of experiments, I satisfied myself by the careful analysis of a specimen of the gas from stagnant water, for which I was indebted to Mr. Dalton, that it really has the properties which have been ascribed to it by him as characteristic; and in 1807 I found precisely the same characters in the fire-damp of coal-mines *. Dr. Thomson, also, from experiments in $1811+$, on the gas from stagnant water, and Sir Humphry Davy $t$, from the analysis of the fire-damp in 1815 , drew the same conclusions. It is in the power, indeed, of every chemist to investigate for himself the properties and composition of carburetted hydrogen gas, since it may easily be procured in considerable quantity, by stirring the bottom of almost any stagnant pool, especially if composed of clay. During the last summer, I obtained it from a source of this kind, which afforded it in such abundance, that several gallons might have been collected in a few miuutes. This gas I submitted to repeated and most careful examination. It contained $\frac{1}{2} \frac{t}{\sigma}$ th its volume of carbonic acid, but no sulphuretted hydrogen whatever, and no proportion of oxygen gas that could be discovered by attentively testing it with nitrous gas. The results of its combustion with oxygen gas, effected in a Volta's eudiometer in the usual manner, showed that it was contaminated with $\frac{1}{15}$ th its volume of azotic gas. Apart, however, from this, the pure portion, in a great number of trials, required, as nearly as can be expected in experiments of this sort, two volumes of oxygen for combustion, and gave one volume of carbonic acid. Its specific gravity, taken on quantities procured at three several times, varied only from $\cdot 582$ to $\cdot 586$, the mean of which is $\cdot 584$; and this, allowing for $\frac{1}{13}$ th of azotic gas of specific gravity $\cdot 972$, gives 556 for the specific gravity of pure carburetted hydrogen gas, a number which coincides almost exactly with that found by Dr. Thomson §. Since, therefore, the same results have been obtained from the examination of gases similarly collected at distant times and places, there appears to me no reason for refusing to consider carburetted hydrogen gas as a true chemical compound, characterized by perfect uniformity of properties and composition. At the temperature of $60^{\circ}$ Fahrenheit, and under 30 inches pressure, 100 cubical inches must weigh 1695 grains, and be composed (taking the weight of 100 cubic inches of carbonic

* Nicholson's Journal, 8vo. xix. 149.

+ Mem. of the Wernerian Society, i. 506 .

t Phil. Trans. 1816, p.5.

$\S$ Annals of Philosophy, vol. xvi. p 252. 
acid at 46.5 grains, and the charcoal in 100 grains of that acid at $27 \cdot 3$ grains) of

$$
\begin{aligned}
& \text { Grains. Grains. Grains. } \\
& \begin{array}{lllllll}
\text { Hydrogen } & \ldots & 4 \cdot 26 & \ldots & 25 \cdot 13 & \ldots & 33 \cdot 41
\end{array} \\
& \overline{16.95} \quad \overline{100} \quad \overline{133.41}
\end{aligned}
$$

And olefiant gas (giving twice its volume of oxygen by combustion, and weighing $29 \cdot 64$ grains for 100 cubical inches ${ }^{*}$ ) must be constituted of

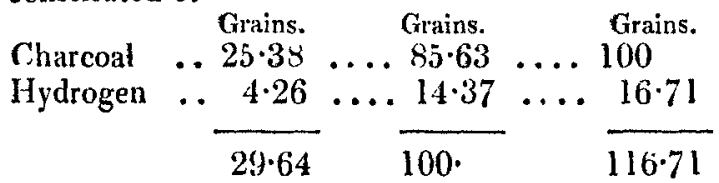

And as 16.7 is to 100 , so very nearly is 1 to 6 , which last number is the weight of the atom of charcoal, as deduced from the constitution of olefiant gas. It is true, that this determination a little exceeds that which is derived from the composition of carbonic acid (viz. 5.65), the atom of oxygen being taken at $7 \cdot 5$. But if 8 be the true number for oxygen, which now seems to be most probable both from experiment and analogy, we shall then find an exact coincidence between the relative weight of the atom of charcoal, as deduced from olefiant gas, and as determined from carbonic acid. Perhaps the true specific gravity of hydrogen gas; on which depend the relative weights of the atoms of hydroger and oxygen, may be fully as correctly ascertained from the composition of carburetted hydrogen, as by direct attempts to weigh so light a fluid. Now, as the hydrogen in 100 cubic inches of hydro-carburet weighs only $4 \cdot 26$ grains, and is equivalent to 200 cubic inches of hydrogen gas, we have $2 \cdot 13$ grains for the weight of 100 cubic inches of hydrogen gas, from which may be deduced .0698 for its specific gravity, that of air being 1 . And if the specific gravity of oxygen gas be $1 \cdot 111$, it will be found that the two volumes of hydrogen, required to saturate one volume of oxygen gas, have as nearly as possible the relative weight of 1 to. 8 .

Were any additional argument necessary to establish the existence of carburetted hydrogen as a distinct species, it might be derived from the action of water on that gas, which, besides being absorbable in a constant proportion, admits of being expelled again by the application of heat, not otherwise changed than in

* I adopt this result of Dr. Thomson from its near coincidence with that of an experiment of my own, on the specific gravity of olefiant gas, published in the Plil. Trans. 1808, p. 293. 
having acquired a small quantity of those gases which are always present in water, and of which it is impossible to deprive it even by long continued boiling.

The process, by which carburetted hydrogen gas is evolved in natural operations, is no doubt the decomposition of water, and admits of being explained on the atomic theory of Mr. Dalton, by supposing two atoms of charcoal to act at once on two atoms of water. One atom of charcoal attracts the two atoms of hydrogen, forming carburetted hydrogen gas, and the other atom of charcoal urites with two atoms of oxygen, constituting carbonic acid. This is illustrated by the annexed figure, in which two atoms of charcoal C.C. are represented as interposed between two atoms of water, each consisting of an atom of hydrogen and an atom of oxygen. Dividing the diagram

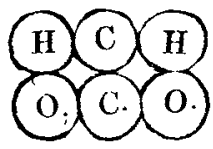
vertically into three parts, we have the original substances; and separating it horizontally, we obtain the two new compounds. This theoretical view of the subject is confirmed by the fact, that the carburetted hydrogen, formed at the bottom of stagnant pools, is never accompanied by carbonic oxide, but always by carbonic acid, the full quantity of which is prevented from appearing, in consequence of the absorption of a great part of it by the mass of water, under which the changes are taking place.

Being provided with such an abundant supply of carburetted lhydrogen, I availed myself of it to examine the mutual action of that gas and chlorine on each other, principally with a view to ascertain, how far reliance may be placed on the latter as an instrument in the analysis of mixed combustible gases. This is a part of the subject that was first investigated, though with a different view, by Mr. Cruickshank*. He observed that a mixture of chlorine with hydrogen, carburetted hydrogen, or carbonic oxide, in certain proportions, kept in a bottle entirely filled with tine mixture, and furnished with an air-tight stopper, did not exhibit any immediate action, but that in twenty-four hours, on withdrawing the stopper, the fluid immediately rushed in, and filled most of the space originally occupied by the gases. But he was not aware of the influence of light on these changes, which was discovered about the same time by Gay Lussac $f$ and by Dalton $f$. It does not, however, appear to have been ascertained by either of them, whether the complete exclusion of light prevents any degree of action of chlorine and carburetted hydrogen on each other. I mixed, therefore, those two gases in different proportions in well stopped vials, which were completely filled

* Nicholson's Journal, 4to. v. 202.

+ Mem. de la Soc. d'Areueil, ii. 349.

$\mp$ New System of Chemieal Philosophy, p. 300. 
with the mixture, and covered by opake cases. When the stoppers were removed under water, at various intervals after the mixture, from a few minutes to 39 days, no diminution whatever of volume was found to have taken place; and after having removed the chlorine by liquid potash, the carburetted hydrogen gas gave the usual products of carbonic acid, and consumed the usual proportion of oxygen. Mixtures also of hydrogen and chlorine, and of carburetted hydrogen and chlorine, standing over water in graduated tubes, which were shaded by opake covers, sustained no loss of bulk, except what arose from the absorption of chlorine by the water, the combustible gas remaining wholly unaltered. It may be considered, therefore, as quite essential to the mutual agency of these gases, that they should be suljected to the infuence of light. But it is not necessary that the direct rays of the sun should fall on the mixture, the light of a dull and cloudy day being fully adequate to the effect. On a day of this sort, I filled several stoppered vials, graduated into hundredths of a cubic inch, with a nixture of 30 volumes of carburetted bydrogen with from 80 to 90 of chlorine, and utcovering them all at the same moment, exposed them to the feeble light which was then abroad. By exposure of one of the vials during half a minute, no diminution of volume was found to have been effected; another vial, opened under water when one minute had elapsed, showed an absorption of five parts; a third in two minutes had lost fifteen parts; a fourth in four minutes 25 parts ; and a fifth, opened in five minutes, contained only 50 volumes out of the original 110 .

The products, resulting from the contact of carburetted hydrogen and chlorine, under circumstances favourable to their mutual action, have been described by Mr. Cruickshank, with whose experience on this point my own entirely agrees. When rather more than four volumes of chlorine are kept in mixture with one volume of gas from stagnant water, the products are muriatic acid gas, and a volume of carbunic acid equivalent to that of the pure carburetted hydrogen; and this, whether the mixture be exposed to direct or indirect solar light; the only difference being that the less intense the light, the more slowly is the effect produced. When less than four volumes of chlorine are employed, the residue consists of muriatic and carbonic acids, carbonic oxide, and undecomposed carburetted hydrogen, the proportions of the two last increasing as, within certain limits, we reduce the relative quantity of chlorine. These changes were ascertained, both by Dr. Davy and the late Dr. Murray, to depend cn the presence of moisture, which is unavoidably introduced in the common mode of operating; for when the gases, first perfectly dried, were mixed in an exhausted glass vessel, and exposed even to the direct rays of the sun, no mutual aetion was 
found to ensue. In the theory of these changes there is, it must be confessed, a little uncertainty. Does the chlorine, it may be asked, act simultaneously on the bydrogen of water, and on that of the combustible gas; or does it decompose water only? The former view of the subject appears to me most probable, because, if the chlorine acted on water only, free hydrogen would be evolved from that portion of the hydro-carburet which abandons its charcoal to the oxygen of the water; which is not consistent with experience. When it is required to form carbonic acid, four volumes of chlorine must be used for the decomposition of each volume of carburetted hydrogen. In this case, two atoms of chlorine unite with the two atoms of hydrogen existing in the combustible gas, and the two other atoms of chlorine with the two atoms of hydrogen from the water. But to convert carburetted hydrogen into carbonic oxide, three atoms of chlorine are sufficient, two of which are employed as in the first case, and the third is expended in saturating the hydrogen of one atom of water, which supplies to the charcoal an atom of oxygen for the formation of carbonic oxide. Calculating in the same manner, we shall find, also, that three atoms of chlorine are adequate to convert one atom of carbonic oxide into carbonic acid.

The facts which have been stated sufficiently prove; that chlorine cannot be employed as a means of correctly analysing mixtures of olefiant gas, either with hydrogen or with carburetted hydrogen, if light be admitted, even though of feeble intensity, and for the short interval during which such an experiment may be expected to continue: and they explain that uncertainty as to the results of analyses of mixed gases made in this way, which was first remarked by Mr. Farraday*, and subsequently by myself $\uparrow$. Chlorine becomes, hrowever, a most usefu! agent in separating olefiant gas from such mixtures, provided light be entirely excluded during its operation, as I have found by subjecting to its action mixtures of those gases with known proportions of olefiant gas. In these analytical experiments, I admitted into a graduated tube standing over water, a volume of chlorine exceeding by about one half what was known to be sufficient, and noted its bulk when actually in the tube, which was immediately shaded by an opake cover. A measured quantity of the mixture was then passed up, and in about ten minutes the outer cover was cautiously lifted, till the surface of the water appeared. The diminution of volume. thus ascertained, divided by 2 , was found to give pretty correctly the quantity of defiant gas known to be contained in the mixture. But the greatest precision was attained by waiting fifteen

* Journal of Science, \&c. vi. 358.

+ Manchester Memoirs, New.Series, vol. ii.

Vol. 58. No.280. Aug. 1521 .

ture. 


\section{On the aëriform Compounds of Charcoal and Hydrogen.}

or twenty minutes, and then quickly washing the remaining gas with dilute solution of potash, in order to remove the excess of chlorine. From the volume of the residuary gas, it was necessary to deduct the amount of impurity previously ascertained to exist in the chlorine; and the remainder, taken from the volume of mixed gases which had been operated on, showed how much olefiant gas had been condensed by the chlorine. When very narrow tubes were employed, and the column of gases mixed with chlorine was of considerable length, a longer continuance of the experiment was found necessary, and the gases were suffered to remain in contnet during an hour or more. In this way it was ascertained, that olefiant gas may be accurately separated by chlorine from hydrogen, carburetted hydrogen, or carbonic oxide gases, or from mixtures of two or more of those gases, which are left quite unchanged in volume and in chemical properties, when light has been carefully excluded from the mixture.

This property of chlorine is the foundation of a fresh analysis, to which I have thought it expedient to submit the gases from coal and oil, in order to decide what aëriform fluids remain after the separation of that portion which is condensible by chlorine; -whether the residue consists, as I have heretofore maintainel, of carburetted hydrogen chiefly, with variable proportions of hydrogen and carbonic oxide; or whether, according to the new view of the subject, it consists of hydrogen gas only.

In the experiments made for this purpose, I operated generally on from 60 to 80 cubic inches of oil gas or coal gas, assaying a small specimen first, as a guide to the quantity of chlorine which it would be necessary to employ. The volume of cllorine thus found to be requisite, and about half as much more, was passed into an air receiver standing over water, and completely shaded by an opake cover which was fitted over it. The oil or coal gas was then added by degrees, if much condensation was expected, because in that case a considerable increase of temperature would have been produced by the sudden admixture of large quantities; or at once, if only a moderate action had been indicated by the previous assay. The mixture was allowed to stand, completely guarded from the light, during 30 or 40 minutes, or even longer, and the residue was expeditiously washed with liquid potash, and a small portion again assayed, to ascertain that the action of the chlorine was complete. The specific gravity of the washed gas was then carefully taken, that of the entire gas having been previously determined: and the results of its combustion with oxygen examined, and compared with those of the gas in its original state.

[The Continuation of this $\mathrm{P}_{\text {aper, }}$ containing Experiments on the Gas from Oil and from Coal, ix our next.]

XVIII. On 